\title{
Hétérogénéité des Préférences pour les Attributs Fourragers en Afrique de l'Ouest : Une Analyse par les Prix Hédoniques Entre le Bénin et le Burkina Faso
}

\author{
Innocent Adédédji Labiyi \\ Laboratoire d'Analyses et de Recherches sur les Dynamiques Economique \\ et Sociale (LARDES), Faculté d'Agronomie, \\ Université de Parakou, Parakou, Bénin \\ Souleymane Ouedraogo \\ Institut de l'Environnement et de Recherches Agricoles (INERA), \\ Burkina Faso \\ Centre Régional AGRHYMET, CILSS, Niamey, Niger \\ Hamadé Sigue \\ Institut de l'Environnement et de Recherches Agricoles (INERA), \\ Fada N'Gourma, Burkina Faso \\ Rosaine Nérice Yegbemey \\ Jacob Afouda Yabi \\ Laboratoire d'Analyses et de Recherches sur les Dynamiques Economique \\ et Sociale (LARDES), Faculté d'Agronomie, \\ Université de Parakou, Parakou, Bénin
}

Doi:10.19044/esj.2019.v15n16p142 URL:http://dx.doi.org/10.19044/esj.2019.v15n16p142

Résumé

En Afrique de l'Ouest et notamment au Sahel, des marchés d'aliments fourragers se développent afin de satisfaire les besoins croissants en fourrages des éleveurs. La présente étude examine les attitudes des éleveurs du bétail à payer pour les attributs fourragers en fonction de leurs préférences respectives sur les marchés de fourrages du Bénin et du Burkina Faso. Dans cette optique, une enquête a été conduite auprès de 275 acheteurs (éleveurs) de fourrages choisis de façon aléatoire stratifiée sur plusieurs sites dans cinq (05) marchés au Bénin et deux (02) marchés au Burkina Faso. Ce faisant, une base de données primaires de 794 observations, suivant les types fourragers achetés par les éleveurs, a été constituée. Pour cela, la méthode de prix hédoniques a été appliquée aux données collectées. Les résultats montrent que le prix des fourrages au Bénin est positivement et significativement influencé par les herbacées vertes, les foins, les fanes (de niébé, d'arachide et du soja) avec des prix implicites positifs et sont plus préférées par les éleveurs. Au Burkina 
Faso, le tourteau du coton et le son du maïs influencent positivement et significativement le prix des fourrages avec une préférence prioritaire dans l'alimentation du bétail. Dans l'ensemble des deux pays, les éleveurs consentent à payer des prix complémentaires lorsqu'il s'agit des concentrées (tourteau du coton, le son du maïs, le son du soja), des résidus de récoltes (la fane du soja, la fane du niébé) et le foin. Ces résultats offrent des possibilités de choix aux différents acteurs du marché pour une meilleure adéquation entre offre et demande en fourrages afin d'en garantir un meilleur approvisionnement pour l'élevage du bétail et une meilleure sécurité alimentaire en Afrique de l'ouest.

Mots-clés: Bénin et Burkina Faso, marchés de fourrages, demande, consentement à payer, prix hédoniques.

\section{Heterogeneity of Feedstuff's Attributes Preferences in West Africa: An Analysis by Hedonic Prices between Benin and Burkina Faso}

\section{Innocent Adédédji Labiyi}

Laboratoire d'Analyses et de Recherches sur les Dynamiques Economique et Sociale (LARDES), Faculté d'Agronomie,

Université de Parakou, Parakou, Bénin

\section{Souleymane Ouedraogo}

Institut de l'Environnement et de Recherches Agricoles (INERA),

Burkina Faso

Centre Régional AGRHYMET, CILSS, Niamey, Niger

\section{Hamadé Sigue}

Institut de l'Environnement et de Recherches Agricoles (INERA),

Fada N'Gourma, Burkina Faso

Rosaine Nérice Yegbemey

Jacob Afouda Yabi

Laboratoire d'Analyses et de Recherches sur les Dynamiques Economique et Sociale (LARDES), Faculté d'Agronomie,

Université de Parakou, Parakou, Bénin 


\section{Abstract}

In West Africa, particularly in the Sahel, feedstuffs markets are developing to meet the growing needs of pastoralists for forage. This study examines the attitudes of livestock farmers to pay for forage attributes according to their respective preferences in the forage markets of Benin and Burkina Faso. In this context, a survey was conducted in five (05) Benin markets and two (02) Burkina Faso markets with 275 feedstuffs buyers selected randomly stratified on several sites. In doing so, a primary database of 794 observations, depending on the forage types purchased by the farmers, was established. For this, the hedonic price method was applied to the collected data. The results show that the price of fodder in Benin is positively and significantly influenced by herbs, hay, haulm (cowpea, peanut and soybean) with positive implicit prices and are more preferred by farmers. In Burkina Faso, Cotton seed cake and corn bran have a positive and significant influence on the price of fodder, with priority given to animal feed. In both countries, farmers agree to pay additional prices for concentrates (Cotton seed cake, corn bran, soybean cake), crop residues (soybean, cowpea haulm) and hay. These results offer opportunities for choice among the different market players for a better match between supply and demand for fodder to ensure a better supply for livestock breeding and better food security in West Africa.

Keywords: Benin and Burkina Faso, feedstuffs' markets, willingness to pay, demand, hedonic prices

\section{Introduction}

Dans de nombreux pays de l'Afrique sahélienne, l'élevage occupe une place centrale et un sous-secteur important de l'agriculture comptant pour $40 \%$ de la production agricole mondiale (FAO, 2016a). Ainsi, il représente une source d'activités et de revenus, une épargne, un facteur de résilience face aux crises alimentaires, mais aussi un référent culturel fort pour plusieurs millions de familles africaines (Thébaud et al., 2018). L’élevage des bovins et ovins en particulier joue alors un rôle crucial dans l'économie ouest-africaine et contribue aux moyens d'existence et à la sécurité alimentaire de près d'un milliard de personnes (FAO, 2015). Il fait ainsi partie de l'un des principaux facteurs d'intégration régionale. Néanmoins, en 2016, la production mondiale de viande n'a globalement augmenté que de $1 \%$ avec un effectif en Afrique de 1'Ouest estimé à plus de 65 millions de bovins, plus de 200 millions d'ovins et caprins et 2,6 millions de camelins (FAO, 2016b).

$\mathrm{Au}$ Bénin, l'élevage représente la seconde activité agricole avec un cheptel national estimé à 3,8 millions de têtes dont estimé à plus de 2,380 millions de bovins et 1,496 millions d'ovins en 2017 (FAO, 2018) en 
contribuant à 2,4\% du PIB. Comme au Benin, le sous-secteur de l'élevage représente la $2^{\text {ème }}$ source de recettes d'exportation après le coton avec 9,647 millions têtes de bovins et 10,1 millions d'ovins au Burkina Faso (FAO, 2018). Par ailleurs, ce sous-secteur emploie plus de $30 \%$ de la population et contribue à environ 12\% du PIB (MRAH, 2014).

Dans les exploitations agricoles familiales du Bénin et du Burkina Faso, l'élevage joue un rôle socio-économique et culturel important. Cependant sa productivité est limitée principalement par des contraintes sanitaires et surtout alimentaires (Koutou et al., 2016). Aussi, dans l'ouest du Burkina Faso, des laiteries se heurtent-elles à un problème d'approvisionnement en lait local, vu sa faible production et saisonnière (Sib et al., 2018). Proche du Burkina Faso, cette situation est de plus en plus ressentie également au Bénin dans plusieurs laiteries installées sur toute l'étendue du territoire national. De ce fait, la couverture des besoins alimentaires du cheptel devient un problème pour chaque éleveur et la faible disponibilité en ressources fourragères est une préoccupation pour les petits éleveurs. La question relative aux disponibilités et aux prix des aliments demeure alors un sujet d'actualité au regard de l'état actuel des circonstances locales, nationales et mondiales (Abdelhakim et Tahar, 2010).

Il convient de souligner que le développement des marchés à fourrages a été également inscrit comme priorité dans la politique agricole du Bénin. Actuellement, la fourniture des marchés en aliments de bétail est assurée par des marchands occasionnels, et reste encore informelle et mal organisée (MAEP, 2011). Dans le même temps, au Burkina Faso, au cours des campagnes agricoles (2012/2013, 2013/2014), 170 à 200 tonnes de semences fourragères de diverses espèces (maïs, sorgho, niébé, mucuna, et soja) ainsi que 180 tonnes de Burkina phosphate ont gracieusement été mises à la disposition des producteurs pour susciter une meilleure production du fourrage et pallier cette insuffisance alimentaire du cheptel (Hazard et al., 2015). Les précédentes études dans la littérature n'ont point fait cas d'une étude de marché de fourrages encore moins d'une analyse de préférences en terme de demande sur les marchés. Toutefois, en matière de commercialisation de fourrage et d'élevage des ruminants, les pays sahéliens comme le Burkina Faso en sont très évolués.

Face à ce constat, l'enjeu de la ressource en protéines animal et végétal apparaît comme crucial chez les éleveurs. Quels sont alors les comportements d'achat des attributs fourragers sur les marchés par les éleveurs? L'objectif de la présente recherche est de mesurer la valeur des différentes caractéristiques fourragères en se basant sur les préférences individuelles des éleveurs de bovins et d'ovins dans les marchés. En prenant le Burkina Faso comme référence en termes de développement des marchés de fourrage, il est important d'identifier les incitations ou motivations appropriées des acheteurs 
de fourrages quant à leur choix des attributs fourragers. L'autre aspect novateur de cette recherche est le fait de prendre deux (02) régions, à savoir le Bénin et le Burkina Faso qui partagent une végétation presque similaire (EstNord), mais des systèmes d'élevage bien différents. La prise en compte des 2 régions favorisera des comparaisons permettant d'aboutir à des contributions de politiques de développement de l'élevage spécifiques à chaque région.

\section{Matériel et méthodes}

\section{Zone de recherche}

La présente étude couvre certains sites de vente de fourrages au Bénin et au Burkina Faso. Le Bénin est situé entièrement dans la zone intertropicale entre $6^{\circ} 30$ et $12^{\circ} 30$ parallèles de latitude nord et couvre une superficie de $112622 \mathrm{~km}^{2}$ (Figure 1). La diversité de son climat permet une grande diversification des ressources végétales et animales à travers le pays (Onibon, 2004). La caractéristique du système d'élevage pratiqué au Bénin est celle de l'élevage semi-sédentaire avec la pratique d'une petite transhumance pendant la saison de culture (pour éviter les dégâts aux cultures) et une grande transhumance pendant la saison sèche. Ces transhumances provoquent des conflits importants entre les éleveurs et les agriculteurs, ce qui rend nécessaire le développement des marchés de fourrages.

Le Burkina Faso a un climat de type soudanien et est situé entre les parallèles de latitude $13^{\circ} 00^{\prime} \mathrm{N}$ et $2^{\circ} 00^{\prime} \mathrm{W}$ (Figure 1). C'est un pays possédant plusieurs zones où la commercialisation des fourrages est une pratique quotidienne et qui occupe de plus en plus d'hommes et de femmes dans les différents secteurs et quartiers de la ville (Sanou et al., 2016). La dynamique de développement de l'élevage de ce pays est semi-intensif utilisant plus de ressources fourragères avec la présence d'un peuplement de $P$. reticulatum et autres, et une grande implication des femmes pauvres dans la récolte des fourrages.

Pour cela, le Burkina-Faso fait partie de la zone de sondage et de référence pour cette étude. 
Figure 1 : Présentation de la zone d'étude

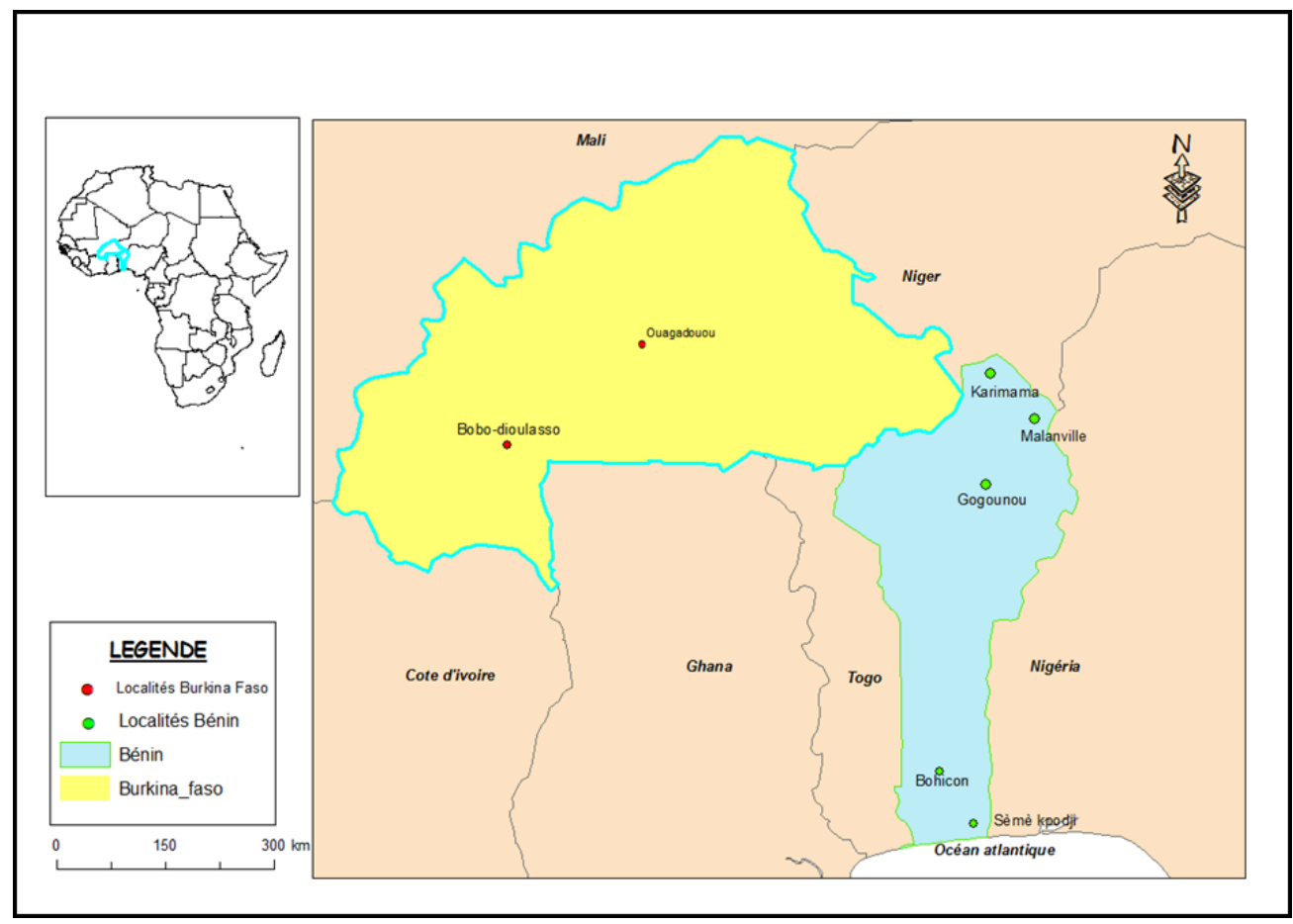

\section{Echantillonnage et base de données}

Les unités de recherche sont les acheteurs de fourrages (éleveurs de bovins et ovins, bouviers et revendeurs) sur différents sites de vente au Bénin et au Burkina Faso.

Sur la base de la pratique de la commercialisation de fourrages, sept (07) grands marchés de fourrages ( 05 au Bénin et 02 au Burkina Faso) repartis sur dix-sept (17) sites de vente ont été choisis dans leur ensemble à raison de six (06) au Bénin et onze (11) au Burkina Faso. Au total, respectivement 155 et 120 acheteurs de fourrages ont été choisis de façon aléatoire stratifiée suivant les sites et de façon raisonnée (selon les caractéristiques fourragères et suivant qu'ils soient éleveurs de bovins ou d'ovins) au Bénin et au Burkina Faso. Les acheteurs rencontrés sur chacun des sites sont systématiquement enquêtés sans remise. Ceci étant, 275 acheteurs de fourrages ont été enquêtés dans l'ensemble des marchés.

Outre les caractéristiques socio-économiques des acheteurs de fourrages sur les marchés, les données collectées portent sur la fréquence d'achat, les types fourragers achetés ainsi que le prix moyen d'achat par botte et par type de fourrage. Un peson électronique a été utilisé pour mesurer les poids de la matière fraîche de fourrage sur les marchés sur trois mois (Septembre, Octobre et Novembre 2018). 
L'analyse quantitative est réalisée à travers le calcul des statistiques descriptives (tableaux de fréquences, moyennes et écarts-types), des tests de Khi deux et de comparaison de moyennes. Le questionnaire électronique est utilisé à travers Kobotoolbox pour collecter les données et le logiciel STATA 13 est utilisé pour analyser les données.

\section{Modèles conceptuel et économétrique utilisés Modèle théorique}

Se basant sur les facteurs de préférences individuelles des consommateurs d'un bien, différentes méthodes d'évaluation non marchande sont utilisées (Travers et al., 2018). Il s'agit généralement des méthodes reposant sur les préférences déclarées (la méthode d'évaluation contingente et la méthode des choix multi-attributs), d'autre part celles reposant sur les préférences observées (la méthode des prix hédonistes). Toutefois, dans la perspective de mesurer l'importance relative des attributs ou caractéristiques d'un bien, seule la méthode des prix hédonistes est adaptée (Lancaster, 1966 et Rosen, 1974) en se basant sur la théorie des attributs. Elle repose sur le constat que les différents biens qui sont échangés sur les marchés sont recherchés uniquement par les quantités de différentes caractéristiques qui les définissent. De ce fait, cette méthode permet de mesurer le consentement à payer marginal pour bénéficier des caractéristiques de fourrages sur le marché.

Pour estimer les modèles hédoniques, les économistes font recours à plusieurs formes fonctionnelles : linéaire, log-linéaire, semi-log, Box-Cox. Dans cet article, il est donc question d'évaluer les différentes valeurs monétaires attribuées par les éleveurs aux caractéristiques de fourrages par la méthode des prix hédoniques à travers la forme semi-log. En effet, cette forme permet d'obtenir les élasticités de chaque variable indépendante par rapport au prix. Elle est aussi moins contraignante et plus explicite que la forme BoxCox. Le modèle se présente comme suit :

$$
\begin{array}{r}
\ln \left(P_{i}\right)=\sum_{j=1}^{J} X_{i j} \beta_{j} \\
+\varepsilon_{i}
\end{array}
$$

Avec

$X_{i j}$ : les caractéristiques des fourrages achetés sur le marché ;

$P_{i}$ : le prix moyen en F CFA par kg des types fourragers sur le marché et, $\beta_{j}$ : l'ensemble des prix marginaux implicites des caractéristiques $\mathrm{j}$, à estimer.

Les variables qualitatives binaires étant les variables explicatives dans le modèle (Equation 1), une estimation g en pourcentage de chaque variable sur le prix est donnée par la formule par l'équation (2) suivante: 
$\mathrm{g}=100\left(e^{x j}-1\right)$

(2)

\section{Estimation du modèle économétrique}

Dans cette étude, la forme fonctionnelle semi-log sur la base du modèle empirique développée par Ladd et Suvannunt (1976), Cavailhès (2005), Terra (2005) et Sodjinou et al. (2015), pour le modèle du prix hédonique, se présente sous la forme :

$$
L n P_{i}=\beta 0+\beta_{j} \sum_{j=1}^{J} F O U_{i j}+\varepsilon_{i}
$$

Où $\beta$ sont des vecteurs de prix implicites marginaux à estimer ; FOUij regroupent les attributs fourragers et sont sous forme non transformée.

Dans le contexte des marchés de fourrages, les caractéristiques extrinsèques des acheteurs n'influencent pas directement le choix des attributs fourragers (Labiyi, 2017). ainsi, les catégories d'attributs fourragers considérés comme variables explicatives sont :

-Les légumineuses fourragères: Herbacées vertes et foin (Ettian et al. 2018 ; Kassa et al., 2018 ; Baumont et al., 2016 ; Sanon et al., 2014) ;

-Les résidus de culture: fanes de légumineuses et tiges de céréales (Archimède et al., 2018 ; Lawal et al., 2018 ; Sanou et al., 2018 ; Delma et al., 2016 ; Montcho et al., 2016 ; Valbuena et al., 2015) ;

-Sous-Produits Agro-Industriels : sons de céréales et tourteaux (Lawal et al., 2018 ; Labiyi, 2017 ; Soumana et al., 2016 ; Montcho et al., 2016 ; Gnanda et al. 2015; Ayantunde et al., 2014 ; Sanon et al., 2011).

Les variables explicatives du prix hédonique du fourrage sont listées dans le Tableau 1.

Par ailleurs, selon Arinloyé (2006), empiriquement le prix marginal implicite ou consentement à payer marginal pour bénéficier de la variation du niveau d'une caractéristique du produit se calcule différemment pour chacune les formes fonctionnelles (linéaire, log-linéaire, semi-log, Box-Cox). Mais, lorsqu'il s'agit du modèle semi-log, le prix implicite d'un changement dans la caractéristique $j$ sur le type fourrager $i$ est le suivant :

$$
\frac{\partial P_{i}}{\partial z_{i j}}=\beta_{j} . P
$$


Tableau 1 : Récapitulatif des variables explicatives du modèle des prix hédoniques

\begin{tabular}{|c|c|c|c|}
\hline Variables & Type & Modalités & Signe attendu \\
\hline Herbacées vertes & Binaire & $1=$ oui $; 0=$ non & $+/-$ \\
\hline Foin & Binaire & $1=$ oui $; 0=$ non & + \\
\hline Fane d'arachide & Binaire & $1=$ oui $; 0=$ non & + \\
\hline Fane du niébé & Binaire & $1=$ oui $; 0=$ non & + \\
\hline Fane du soja & Binaire & $1=$ oui $; 0=$ non & + \\
\hline Fane de la patate douce & Binaire & $1=$ oui $; 0=$ non & + \\
\hline Paille/tige du maïs & Binaire & $1=$ oui $; 0=$ non & - \\
\hline Paille du sorgho & Binaire & $1=$ oui $; 0=$ non & - \\
\hline Paille du riz & Binaire & $1=$ oui $; 0=$ non & - \\
\hline Son du soja & Binaire & $1=$ oui $; 0=$ non & + \\
\hline Son du maïs & Binaire & $1=$ oui $; 0=$ non & + \\
\hline Son du blé & Binaire & $1=$ oui $; 0=$ non & + \\
\hline Tourteaux du coton & Binaire & $1=$ oui $; 0=$ non & + \\
\hline Blocs alimentaires & Binaire & $1=$ oui $; 0=$ non & + \\
\hline
\end{tabular}

Afin de minimiser les erreurs d'estimation (forte multi-colinéarité entre les variables avec une hétéroscédasticité du modèle), la régression linéaire multiple robuste est estimée pour tenir compte de l'hétéroscédasticité avec une correction de White.

\section{Résultats}

\section{Caractéristiques des acheteurs de fourrages sur les marchés au Bénin et au Burkina Faso}

Presque tous les acheteurs de fourrage enquêtés dans les marchés au Bénin et au Burkina Faso sont des hommes (Tableau 2). Très peu d'entre eux ont adhéré à une association ou réseaux d'éleveurs/d'acheteurs de fourrages dans la zone d'étude. La majorité des acheteurs $(70,2 \%)$ sont propriétaires de leur cheptel et presque la moitié conduit leurs animaux en transhumance. De plus, $21,5 \%$ des enquêtés de la zone d'étude ont reçu une éducation formelle (majoritairement du niveau primaire) et $14,9 \%$ d'entre eux sont alphabétisés en langues locales.

En effet, les raisons de l'achat ou du choix d'un type de fourrage pour l'alimentation par les éleveurs enquêtés sont orientées vers : l'embouche (75,3 $\%)$, la production du lait $(11,3 \%)$ et la complémentation $(45,8 \%)$. 
Tableau $2:$ Récapitulatif des variables qualitatives des acheteurs de fourrages

\begin{tabular}{cccccc}
\hline Variables (\%) & Modalités & Bénin & Burkina Faso & Ensemble & Test de Khi-2 \\
\hline Sexe de & Femme & 1,7 & 1,9 & 1,8 & $\chi^{2}=0,027 ; \mathrm{P}=0,869$ \\
l'acheteur & Homme & 98,3 & 98,1 & 98,2 & \\
Appartenance à un groupement & 23,3 & 11,6 & 16,7 & $\chi^{2}=6,670 ; \mathrm{P}=0,010$ \\
Pratique de la transhumance & 50 & 46,7 & 47,1 & $\chi^{2}=0,063 ; \mathrm{p}=0,802$ \\
Education formelle de l'acheteur & 26,7 & 17,4 & 21,5 & $\chi^{2}=3,432 ; \mathrm{P}=0,064$ \\
Alphabétisation en langues locales & 14,2 & 15,5 & 14,9 & $\chi^{2}=0,093 ; \mathrm{P}=0,761$ \\
Mode & Bouvier & 13,3 & 17,4 & 15,6 & $\chi^{2}=1,132 ; \mathrm{P}=0,568$ \\
d'acquisition du & Propriétaire & 73,3 & 67,7 & 70,2 & \\
bétail & Bouvier et propriétaire & 13,3 & 14,8 & 14,2 & \\
Raisons d'achat & Embouche & 70,8 & 78,7 & 75,3 & $\chi^{2}=2,254 ; \mathrm{P}=0,133$ \\
du fourrage & Complémentation & 42,5 & 48,4 & 45,8 & $\chi^{2}=0,944 ; \mathrm{P}=0,198$ \\
& Production du lait & 4,2 & 16,8 & 11,3 & $\chi^{2}=10,749 ; \mathrm{P}=0,001$ \\
\hline
\end{tabular}

Dans l'ensemble, l'âge moyen des acheteurs de fourrages est de 41,91 $( \pm 12,37)$ ans (Tableau 3) avec une expérience moyenne de 13,96 $( \pm 9,050)$ dans l'élevage. Les acheteurs béninois enquêtés sont moins expérimentés en élevage $(\mathrm{p}<0,01)$. Dans les deux pays, la taille du cheptel moyen en bovins est estimée à $22,52( \pm 39,643)$ têtes avec une variation de 1 à 250 têtes. En ce qui concerne les petits ruminants, le cheptel moyen est estimé à 13,19 $( \pm 14,12)$ têtes avec une variation de 1 à 100 ovins. Ces cheptels varient significativement selon les pays $(\mathrm{p}<0,01)$. Le revenu total annuel issu de l'élevage dans la zone d'étude est de 1533677 ( \pm 1352 173,03) FCFA et ceci varie significativement d'un pays à un autre $(\mathrm{p}<0,01)$.

Tableau 3 : Récapitulatif des variables quantitatives des acheteurs de fourrages

\begin{tabular}{|c|c|c|c|c|}
\hline Variables & Zones d'étude & Moyenne & Ecart-type & Test t-Student \\
\hline \multirow{3}{*}{ Age de l'acheteur } & Burkina Faso & 41,08 & 11,913 & \multirow{3}{*}{$\begin{array}{c}\mathrm{t}=-0,969 ; \mathrm{ddl}=273 ; \\
\mathrm{p}=0,333\end{array}$} \\
\hline & Bénin & 42,54 & 12,727 & \\
\hline & Ensemble & 41,91 & 12,377 & \\
\hline \multirow{3}{*}{ Expérience dans l'élevage } & Burkina Faso & 16,50 & 9,489 & \multirow{3}{*}{$\begin{array}{c}\mathrm{t}=-5,589 ; \mathrm{ddl}=273 ; \\
\mathrm{p}=0,000\end{array}$} \\
\hline & Bénin & 10,67 & 7,259 & \\
\hline & Ensemble & 13,96 & 9,050 & \\
\hline \multirow{3}{*}{ Revenu total annuel d'élevage } & Burkina Faso & 1891571,52 & 1781775,462 & \multirow{3}{*}{$\begin{array}{c}\mathrm{t}=5,388 ; \mathrm{ddl}=273 ; \\
\mathrm{p}=0,000\end{array}$} \\
\hline & Bénin & 1153080,566 & 934574,19 & \\
\hline & Ensemble & 1533677,989 & 1352173,03 & \\
\hline \multirow{3}{*}{ Nombre de tête de bovins } & Burkina Faso & 33,03 & 49,464 & \multirow{3}{*}{$\begin{array}{c}\mathrm{t}=-5,090 ; \mathrm{ddl}=273 ; \\
\mathrm{p}=0,000\end{array}$} \\
\hline & Bénin & 9,25 & 12,639 & \\
\hline & Ensemble & 22,52 & 39,643 & \\
\hline \multirow{3}{*}{$\begin{array}{l}\text { Nombre de têtes de petits } \\
\text { ruminants }\end{array}$} & Burkina Faso & 16,29 & 14,399 & \multirow{3}{*}{$\begin{array}{c}\mathrm{t}=-4,142 ; \mathrm{ddl}=273 ; \\
\mathrm{p}=0,000\end{array}$} \\
\hline & Bénin & 9,29 & 12,795 & \\
\hline & Ensemble & 13,19 & 14,125 & \\
\hline
\end{tabular}

\section{Prix de fourrages vendus dans les marchés à bétail au Bénin et au Burkina Faso}

Dans les marchés de la zone d'étude, les différents fourrages les plus commercialisés et achetés sont majoritairement des aliments grossiers, les herbacées naturelles $(83,6 \%)$ telles que des graminées, des légumineuses et les foins. En plus de ces graminées et des légumineuses, s'ajoutent les résidus de 
cultures $(69,1 \%)$ et les Sous-Produits de transformation Agro-Industriels $(50,9 \%)$.

Le prix des fourrages vendus sur les marchés varie d'un type fourrager à un autre et aussi d'un site de vente à un autre dans la zone d'étude. Ainsi, dans les zones urbaines qui abritent d'ailleurs les marchés terminaux ou de consommation du bétail, les prix des fourrages sont élevés que dans les zones rurales (marché de regroupement ou de production).

Dans l'ensemble, les prix des herbacées naturelles sont très élevés et varient très significativement sur les sites du Bénin qu'au Burkina Faso. Par contre, les légumineux issus des résidus de récolte (fanes surtout) et les concentrées (sous- produits agro-alimentaires) sont vendus plus chers sur les sites de vente du Burkina Faso dans la zone d'étude.

\section{Facteurs déterminants du prix des fourrages sur les marchés} Préférences des acheteurs de fourrages sur les marchés du Bénin

L'analyse des résultats du tableau 4 montre que le modèle de la régression des prix hédoniques des types fourragers présente une excellente significativité globale au seuil de $1 \%(\mathrm{p}<0,01)$ avec un assez bon pouvoir explicatif $\left(\mathrm{R}^{2}=0,4709\right)$. Les résultats de l'estimation témoignent de la significativité des attributs fourragers: herbacées vertes, foin; fanes d'arachide, de niébé, du soja, pailles de maïs, du sorgho et du riz, sons du soja et du blé ; et épluchures de tubercules (Tableau 4). Dans l'ensemble, le prix $\mathrm{du}$ fourrage répond plus proportionnel aux différentes variations de ces attributs. Ce qui est justifié par les forts coefficients estimés dans le modèle.

\section{Herbacées naturelles : fourrages verts et foins}

Les foins et les herbacées vertes ont un effet positif sur le prix de vente des fourrages sur les sites du Bénin avec des élasticités prix respectivement de 0,60 et 0,183 . Les valeurs implicites 103,21 FCFA et 18,93 FCFA associées respectivement à ces caractéristiques du fourrage représentent leur prix hédonique. Ainsi, les éleveurs seraient disposés à payer respectivement 103,21 FCFA et 18,93 FCFA pour s'acheter respectivement $1 \mathrm{~kg}$ de foin et des herbacées vertes sur les sites de vente du Bénin.

Résidus de récolte : Fanes (d'arachide, du niébé et du soja) et pailles (du maïs, du sorgho, et du riz)

Sur l'ensemble des sites du Bénin, les légumineuses (fanes d'arachide, du niébé et du soja) ont un effet positif sur le prix de vente des fourrages avec des élasticités associées à chacun des types fourragers positives et respectivement égales à 0,$585 ; 0,911$ et 0,835 . Dans ce cas, les acheteurs sont prêts à acheter chacun de ces types fourragers au marché respectivement à 104,94 FCFA, 207,93 FCFA et 160,47 FCFA par kilogramme. 
Contrairement aux légumineuses, les pailles issues des résidus de récolte (du maïs, du sorgho, et du riz) présentent des élasticités négatives. Dans ce point de vue, les éleveurs sont prêts à acheter ces types de fourrages au marché avec des réductions respectives de 25,448 FCFA, 36,156 FCFA et 24,664 FCFA sur le kilogramme du prix de vente.

\section{Sous-Produits Agro-Industriels : Sons du soja, du maïs, du blé et les épluchures de tubercules}

L'élasticité prix du son du soja est positive $(0,373)$ et induit une valeur implicite de $46 \mathrm{~F} \mathrm{CFA} / \mathrm{Kg}$. Par conséquent, les acheteurs consentent à payer une augmentation de 46,16 FCFA sur le kilogramme du son du soja dans les marchés du Bénin. Par contre, les élasticités prix associées aux sons du maïs, du blé et les épluchures de tubercules sont négatives $(-0,132,-0,303$ et $-0,689$ respectivement) induisant ainsi des prix implicites négatifs.

\section{Préférences des acheteurs de fourrages sur les marchés du Burkina Faso}

L'analyse des résultats (Tableau 4) montre que le modèle de la régression des prix hédoniques des types fourragers au Burkina Faso est globalement significatif au seuil de $1 \%(\mathrm{p}<0,01)$ avec un pouvoir explicatif de 0,5084 . Les résultats de l'estimation témoignent de la significativité des attributs fourragers : herbacées vertes, paille sorgho, son maïs, tourteau du coton au seuil de 1\%. Dans l'ensemble, le prix du fourrage répond plus proportionnel aux différentes variations de ces attributs.

\section{Herbacées naturelles : fourrages verts}

$\mathrm{Au}$ Burkina Faso, les fourrages verts présentent une élasticité prix négative de $-0,360$ avec ainsi un prix implicite de $-21,70$ FCFA. Une augmentation du prix en état actuel de cette herbacée naturelle fait renoncer son choix chez l'acheteur sur le marché.

\section{Résidus de récolte : Pailles du sorgho}

Comme au niveau des herbacées naturelles, au niveau des pailles du sorgho, la valeur implicite à ce type de fourrage est négative $(-33,20$ FCFA) et son élasticité varie plus que proportionnellement au prix.

\section{Sous-Produits Agro-Industriels : Tourteau du coton et son du maïs}

L'analyse des coefficients de la régression révèle que les élasticités associées à chacun des types fourragers sont positives et respectivement égales à 1,407 et 0,595 pour le son du maïs et les tourteaux du coton. Les prix hédoniques au kilogramme associés à chacun sont respectivement de 514,19 FCFA pour le son du maïs et de 104,218 FCFA pour le tourteau du coton. 


\section{Préférences des acheteurs de fourrages sur les marchés au Bénin et au Burkina Faso}

Le modèle de régression de la fonction des prix hédoniques des types fourragers présente significativité globale au seuil de $1 \%(\mathrm{p}<0,01)$ avec un assez bon pouvoir explicatif $\left(\mathrm{R}^{2}=0,2777\right)$. Les attributs fourragers : herbacées vertes et foin ; les fanes du niébé, du soja, paille/tige maïs, paille du sorgho, paille du riz; les sons du maïs, les sons du blé et du soja, tourteaux du coton et épluchures de tubercules déterminent significativement le prix d'achat de fourrages sur l'ensemble des marchés (Tableau 4).

\section{Herbacées naturelles : fourrages verts et foins}

Sur les marchés du Bénin et du Burkina Faso, l'élasticité prix du foin est positive $(0,386)$ avec une valeur implicite positive $(60,57$ FCFA) associée à la caractéristique fourragère. Ainsi, les acheteurs seraient disposés à payer 60,57 FCFA pour s'acheter $1 \mathrm{~kg}$ de fourrage. Par contre, l'élasticité prix des fourrages en herbacées vertes est négative $(-0,19)$ sur l'ensemble des marchés.

Résidus de récolte : Fanes (du niébé et du soja), pailles (du maïs, du sorgho et du riz) et épluchures de tubercules

A ce niveau, les élasticités prix des fanes du niébé et du soja sont positives avec des prix implicites respectivement de 74 FCFA et 137 FCFA. Ces fanes sont plus appréciées par les éleveurs pour l'alimentation bovine à cause de leurs valeurs nutritives et de leur apport en vitamines au bétail. Par contre, les pailles du riz $(-0,523)$, du Sorgho $(-1,290)$ et du maïs $(-0,645)$ ainsi que les épluchures de tubercules $(-0,812)$ présentent des élasticités négatives.

\section{Sous-Produits Agro-Industriels : Tourteau du coton, sons du soja, du maïs et du blé}

Les élasticités prix associées au tourteau du coton, aux sons du soja et $\mathrm{du}$ maïs sont positives et respectivement égales à 0,$367 ; 0,250$ et 0,544 (Tableau 5). Du coup, pour une augmentation des prix de ces types fourragers, les éleveurs sont prêts à les acheter respectivement à 59 FCFA, 31 FCFA et 122 FCFA par kilogramme. Par contre, dans l'ensemble le son du blé présente une élasticité négative $(-0,456)$ avec une négative valeur implicite $(-48,66$ FCFA). De même, malgré sa richesse, les acheteurs sont moins sensibles à la variation des blocs alimentaires. 
Tableau 4 : Récapitulatif des modèles de régression des déterminants du prix de fourrage sur les marchés dans la zone d'étude

\begin{tabular}{|c|c|c|c|c|c|c|}
\hline \multirow[t]{2}{*}{ Paramètres } & \multicolumn{2}{|c|}{ Bénin } & \multicolumn{2}{|c|}{ Burkina Faso } & \multicolumn{2}{|c|}{ Ensemble des marchés } \\
\hline & Coefficients & Prix hédoniques & Coefficients & Prix hédoniques & Coefficients & Prix hédoniques \\
\hline Herbacées vertes & $0,183(0,110)^{*}$ & 18,933 & $-0,360(0,089)^{* * *}$ & $-21,700$ & $-0,1939(0,087)^{* *}$ & $-15,802$ \\
\hline Foin & $0,606(0,136) * * *$ & 103,219 & --- & --- & $0,386(0,118)^{* * * *}$ & 60,573 \\
\hline Fane d'arachide & $0,585(0,128) * * *$ & 104,949 & --- & --- & $0,149(0,108)$ & 20,354 \\
\hline Fane du niébé & $0,911(0,117)^{* * *}$ & 207,935 & $0,183(0,118)$ & 22,512 & $0,427(0,100)^{* * * *}$ & 74,027 \\
\hline Fane du soja & $0,835(0,147) * * *$ & 160,471 & --- & --- & $0,713(0,133) * * *$ & 136,913 \\
\hline Fane de la patate douce & -- & --- & $0,237(0,327)$ & 30,627 &.-- & --- \\
\hline Paille/tige du maïs & $-0,532(0,130) * * *$ & $-25,448$ & --- & --- & $-0,645(0,104) * * *$ & $-31,143$ \\
\hline Paille du sorgho & $-1,203(0,306)^{* * *}$ & $-36,156$ & $-1,135(0,207)^{* * *}$ & $-33,209$ & $-1,290(0,218) * * *$ & $-38,429$ \\
\hline Paille du riz & $-0,387(0,185) * *$ & $-24,664$ & --- & --- & $-0,523(0,161) * * *$ & $-32,630$ \\
\hline Son du soja & $0,373(0,124) * * *$ & 46,165 & --- & --- & $0,250(0,107)^{* *}$ & 30,992 \\
\hline Son du maïs & $-0,132(0,120)$ & $-10,285$ & $1,407(0,102) * * *$ & 514,195 & $0,544(0,129)^{* * * *}$ & 121,502 \\
\hline Son du blé & $-0,303(0,166)^{*}$ & $-32,3487$ & --- & --- & $-0,456(0,137) * * *$ & $-48,663$ \\
\hline Tourteaux du coton & --- & -- & $0,595(0,120)^{* * *}$ & 104,218 & $0,367(0,130)^{* * *}$ & 59,574 \\
\hline Blocs alimentaires & --- & --- & --- & --- & $0,551(0,455)$ & 84,817 \\
\hline Epluchures de tubercules & $-0,689(0,151)^{* * *}$ & $-30,209$ & --- & --- & $-0,812(0,137) * * *$ & $-35,582$ \\
\hline Constantes & $4,396(0,103)^{* * *}$ & --- & $4,435(0,086)^{* * *}$ & --- & $4,519(0,082)^{* * *}$ & --- \\
\hline \multicolumn{7}{|c|}{ Résumés des modèles } \\
\hline \multicolumn{3}{|c|}{ Nombre d'observations } & 351 & & \multicolumn{2}{|l|}{794} \\
\hline Valeur de F & \multicolumn{2}{|c|}{$(12,430)=40,43$} & $(6,344)=174,98$ & & \multicolumn{2}{|c|}{$(14,779)=32,22$} \\
\hline Prob $>F$ & \multicolumn{2}{|c|}{0,000} & 0,0000 & & \multicolumn{2}{|l|}{0,0000} \\
\hline R-squared & \multicolumn{2}{|c|}{0,4709} & 0,5084 & & \multicolumn{2}{|l|}{0,2777} \\
\hline
\end{tabular}

$* * *=$ significatif à $1 \% ; * *=$ significatif à $5 \%, *=$ significatif à $10 \%$; les valeurs entre parenthèses sont les erreur-types 


\section{Discussion}

Des pays sahéliens vers ceux côtiers en Afrique subsaharienne, les systèmes d'élevage sont soutenus par un système d'alimentation adéquat à travers une disponibilité des aliments gage d'une complémentation ou d'embouche. Les résultats de cette étude montrent que plusieurs attributs fourragers sont vendus sur les sites de vente de fourrages au Bénin et au Burkina Faso. Il s'agit des groupes fourragers : herbacées naturelles, résidus de récolte et les Sous-Produits Agro-Industriels. Au sein de chaque groupe, plusieurs types fourragers sont vendus et varient d'un site de vente à un autre et d'un pays à un autre. De même, les prix du fourrage varient significativement selon les types fourragers et les pays. Globalement, les légumineuses fourragères (toute nature) sont plus prisées sur les marchés avec une hausse considérable sur les marchés du Burkina Faso. Dans ce sens, Dan gomma et al. (2000), Sanou et al. (2011) et Maman (2014) ont estimé le prix de différents types fourragers de rationnement dans les opérations d'embouche et de complémentation dans les différents marchés à bétail en Afrique de l'Ouest. Ils ont trouvé que la qualité du fourrage est un facteur déterminant son prix de vente.

Au Burkina Faso, les prix des fourrages connaissent des augmentations importantes sur les principaux marchés de fourrages. Les fanes de légumineuses et les sous-produits de transformation agro-industriels issus des légumineuses prennent de plus en plus de valeur au fur et à mesure que l'on passe de la saison de pluie à la saison sèche et caractérisée par des différents évènements qui se réalisent ou s'annoncent avec la montée des prix des fourrages. Pour Archimède et al. (2018), à comparer aux céréales, les protéines des légumineuses graines sont généralement riches en lysine et donc très adaptés aux ruminants. Les résultats ont également montré que les aliments concentrés (son du maïs et le tourteau de coton) sont plus préférés par les acheteurs de fourrages au Burkina Faso. Cette montée de prix est alarmante dans les zones sahéliennes (Fode, 2010 et FAO, 2014) dont le Burkina Faso. De plus, du point de vue nutritionnel, ces types de fourrage sont caractérisés par leur valeur nutritive et leur digestibilité. Pour Singh et al. (2013), les marchés fourragers sont importants pour les communautés qui ont une capacité limitée à produire leur propre fourrage, mais ont besoin de fourrages de qualité à des prix raisonnables.

Par contre, sur les marchés de fourrages au Bénin, les légumineuses herbacées et les fanes des légumineuses issues des résidus de récolte sont préférées par les acheteurs et sont prêts à s'en approvisionner dans une perspective d'augmentation de leurs prix. Les fanes de légumineuses (niébé, soja ou d'arachide) peuvent avoir une valeur commerciale élevée sur les marches urbaines africains (Delma et al., 2016; Montcho et al., 2016 ; Ayantunde et al., 2014). Ces fanes de légumineuses sont des fourrages de 
qualité qui peuvent être utilisés comme seul fourrage ou supplément à des fourrages pauvres (Archimède et al., 2018). Selon Guérin et Roose (2015), les bovins consomment davantage de pailles de graminées sèches et recherchent avidement les fanes de légumineuses. De même, les tourteaux de soja constituent la source la plus importante de complément protéique, bien que les sous-produits d'origine animale tels que les résidus d'abattoirs et de pêche en soient d'excellentes sources (Cissé, 2015). Valbuena et al. (2015) sont parvenus à la conclusion que les préférences des éleveurs se reflètent dans leurs décisions concernant l'utilisation actuelle des résidus disponibles. Les préférences de ces derniers et les utilisations varient d'un groupe à l'autre et surtout en fonction de la structure de leurs ménages. Toutefois, la fabrication des blocs alimentaires à grande échelle peut être une réponse à la conservation et à l'utilisation efficace des ressources végétales dans une perspective de développement de marchés et de satisfaction des éleveurs.

\section{Conclusion}

En Afrique de l'Ouest, le développement des marchés de fourrage devrait être inscrit comme priorité dans les politiques agricoles et de l'élevage. Cette étude a permis de mieux comprendre les comportements microéconomiques des propriétaires du bétail ainsi que les bouviers sur les marchés dans le choix des fourrages. Les résultats ont montré que globalement les herbacées naturelles, les fanes des légumineuses, le son du soja sont plus préférés avec des prix implicites positifs sur les marchés du Bénin tandis que le son du maïs et le tourteau du coton font la priorité des acheteurs au niveau des marchés du Burkina Faso. En général, le choix et la préférence sont focalisés sur l'objectif de l'élevage. Les résultats obtenus offrent des possibilités de choix de compléments alimentaires aux agro-éleveurs pour un engraissement des ruminants en milieu tropical humide de l'Afrique de l'Ouest. En d'autres termes, l'influence de ces différentes variables sur la production, la consommation et les préférences des éleveurs peut être utilisée comme instruments de politiques agricoles en suggérant la culture des fourrages légumineux et en couplant l'agriculture et l'élevage dans les deux pays pour mieux répondre aux besoins des animaux.

\section{References:}

1. Abdelhakim, S., Tahar, B., 2010. Etude des disponibilités des aliments de bétails dans les régions sahariennes. Cas de la Région du Souf. Revue du chercheur, 10 (1) : 10.

2. Archimède, H., Bastianelli, D., Fanchone, A., Gourdine J-L, Fahrasmane, L., 2018. Aliments protéiques dans les systèmes mixtes intégrés polyculture-élevage en régions tropicales. INRA Prod. Anim 31: 221-236. 
3. Arinloyé, D.A.A. 2006. Analyse des facteurs déterminant la demande du riz au centre et au Sud du Bénin. Thèse d'obtention du diplôme d'ingénieur agronome (FSA/UAC); pp 97.

4. Ayantunde, A.A., Blummel, M., Grings, E., Duncan, A.J., 2014. Prix et qualité des aliments du bétail dans les marchés périurbains du Sahel Ouest africain: étude de cas à Bamako, Mali. Rev. D’élevage Médecine Vét. Pays Trop 67: 13-21.

5. Baumont, R., Bastien, D., Férard, A., Maxin, G., Niderkorn, V., 2016. Les intérêts multiples des légumineuses fourragères pour l'alimentation des ruminants. Fourrages 227, 171-180.

6. Cavailhès, J., 2005. Le prix des attributs du logement. Economie et statistique, Centre National de Ressources Textuelles et Lexicales (CNRTL). $\quad$ pp. 381-382. Site web: http://archive.wikiwix.com/cache/?url=http\%3A\%2F\%2Fwww.cnrtl.fr \%2Fetymologie\%2Ffeurre consulté le 22/09/2017 à $10: 32$

7. Dan Gomma, A, Rupol,. P. 2000. Etude sur la production des ruminants en milieu urbain et périurbain de Niamey, Niger.-Niamey : AD-VSFFPI. $77 \mathrm{p}$.

8. Delma, B.J., Bougouma-Yameogo, V., Nacro, B.H., Vall, E., 2016. Altrop, un calculateur des rations des bovins pour l'accompagnement des projets d'élevage familiaux. International Journal of Biological and Chemical Sciences 10: 966-982.

9. Ettian, M.K., Gbogouri, G.A., Djenontin, J.A., Mensah, G.A., 2018. Évaluation des apports nutritionnels et énergétiques des fourrages verts dans l'alimentation des aulacodes (Thryonomys swinderianus) en Côte d'Ivoire. Rev. Marocaine Sci. Agron. Vét. 6, 99-109.

10. FAO. 2016a. Perspectives agricoles de l'OCDE et de la FAO 2016-2025, Éditions OCDE, Paris, 147p. http://dx.doi.org/10.1787/agr_outlook2016-fr.

11. FAO, 2016b. Consommation de viande. Organisation des Nations Unies pour l'Alimentation et l'Agriculture (FAO), Département de l'Agriculture et de la Protection des Consommateurs. Disponible en ligne : http://www.fao.org/ag/againfo/themes/fr/meat/background.html.

12. FAO. 2018. FAO Stat, http://www.fao.org/faostat/fr/\#data/QA, (consulté le 03/06/2019).

13. FAO. 2014. Résidus agricoles et sous-produits agro-industriels en Afrique de l'ouest: Etat des lieux et perspectives pour l'élevage. Rapport d'étude. Ghana, Accra: FAO.

14. Fode, C.M.S., 2010. Durabilité des systèmes pastoraux et dynamique de la strate herbacée des pâturages de la zone sahélienne du Niger (Afrique de l'ouest). Thèse de doctorat en Sciences de la Nature. Alger : Université des Sciences et Technologies HOUARI BOUMEDIENE. 
15. Guérin, H., Roose, E., 2015. Ingestion, restitution et transfert d'éléments fertilisants aux agro systèmes par les ruminants domestiques en régions semi arides d'Afrique occidentale: points de vue d'un zootechnicien et d'un agro-pédologue. Cirad, UMRSELMET, 18p. http://horizon.documentation.ird.fr/exl-doc/pleins_textes/divers1605/010064966.pdf

16. Hazard, L., Goutiers, V., Moirez-Charron, M-H., Deo M., Martin, G., 2015. Outiller la bio- diversification des systèmes fourragers pour les adapter au changement climatique. Colloque présentant les méthodes et résultats du projet Climagie (métaprogramme ACCAF), Poitiers, France. INRA, $223 \mathrm{p}$.

17. Kassa, K.A.D., Nenonene, A.Y., Tchainiley, L., Koba, K., 2018. Pratiques de la production et d'exploitation des fourrages dans la Région des Plateaux au Togo. Int. J. Biol. Chem. Sci. 12, 1415-1422.

18. Koutou, M., Sangare, M., Havard, M., Toillier, A., Sanogo, L., Thombiano, T., Vodouhe, D.S., 2016. Income source and accompaniment needs of family farms in the cotton growing area of West Burkina Faso. Biotechnologie, Agronomie, Société et Environnement 20, 42-56.

19. Ladd, G., Suvannunt, V., 1976. A model of consumer goods characteristics. Agricultural Economics 58: 504-510.

20. Lancaster, K.J., 1966. A new approach to consumer theory. Journal of Political Economy 74 (2): 132-157.

21. Lawal, A.M., Chaibou, M., Mani, M., Garba, M.M., Gouro, A.S., 2018. Pratiques d'éleveurs et résultats économiques d'élevage dans les exploitations urbaines et périurbaines de Niamey. Int. J. Biol. Chem. Sci. 12, 294-309.

22. Maman, M.S., 2014. Analyse du système de commercialisation du fourrage dans la ville de Niamey (Niger). Mémoire de master, Ecole Inter-états des Sciences et Médecine vétérinaires (EISMV) de Dakar, 41 p.

23. Ministère des Ressources Animales, du Ministère de l'Agriculture, de l'Hydraulique et des Ressources Halieutiques et des représentants des Chambres Régionales d'Agriculture, 2014. Analyse de la filière bétailviande au Burkina Faso. Rapport EASYPol, 167 pages.

24. MINISTERE DE L'AGRICULTURE, DE L'ELEVAGE ET DE LA PECHE (MAEP), DIRECTION DE LA PROGRAMMATION ET DE LA PROSPECTIVE. 2012. Rapport de performance du secteur agricole. 42p. http://www.roppa-afrique.org/IMG/pdf/forma_1_rapport.pdf.

25. Montcho, M., Babatoundén S., Aboh, B.A., Bahini, M.J.D., Chrysostome, A., Mensah, G.A., 2016. Disponibilité, Valeurs Marchande Et Nutritionnelle Des Sous-Produits Agricoles Et 
Agroindustriels Utilisés Dans L'alimentation Des Ruminants Au Benin. European Scientific Journal $33 \quad$ (12):422-441. http://doi:10.19044/esj.2016.v12n33p422

26. Onibon, P., 2004. Accès au marché des produits agricoles. Forum du Centre technique de coopération agricole et rurale (CTA); Bénin, octobre 2004, 58p.

27. Rosen, S., 1974. Hedonic prices and implicit markets: Product differentiation in pure competition. Journal of Political Economy 82 (1): 35-55.

28. Sanon, H.O., Some, S., Obulbiga, M.F., Oubda, F.A., Bamouni, I., 2018. Analyse de la structure et du fonctionnement de la filière fourrage dans les villes de Ouagadougou et Bobo-Dioulasso au Burkina Faso. Int. J. Biol. Chem. Sci. 12, 1247-1259.

29. Sanon, H.O., Drabo, A., Sangare, M., Kiendrebeogo, T., Gomgnibou, A., 2014. Caractérisation des pratiques d'embouche bovine dans l'Ouest du Burkina Faso. International Journal of Biological and Chemical Sciences 8: 536-550.

30. Sanou, K.F., Ouédraogo, S., Nacro, S., Ouédraogo, M., KaboréZoungrana, C., 2016. Durabilité de l'offre et valeur nutritive des fourrages commercialisés en zone urbaine de Bobo-Dioulasso, Burkina Faso. Cahiers Agricultures 25: 15002.

31. Sib, O., Bougouma-Yameogo, V.M., Blanchard, M., Gonzalez-Garcia, E., Vall, E., 2018. Production laitière à l'ouest du Burkina Faso dans un contexte d'émergence de laiteries: diversité des pratiques d'élevage et propositions d'amélioration. Revue d'élevage et de médecine vétérinaire des pays tropicaux 70, 81-91.

32. Singh, K.M., Singh, R.K.P., Jha A.K., Kumar, A., 2013. Fodder market in Bihar: An exploratory study. Economic Affair 58, 357.

33. Sodjinou, E., Henningsen, A., Koudandé, D.O., Biaou, G., Mensah, G.A., 2015. Consumer's preferences for"bicycle poultry" in Benin: Implications for the design of breeding schemes. Revue d'Études en Agriculture et Environnement 96:389-409 doi:10.4074/S196696071500301X.

34. Terra, S., 2005. Guide de données pratiques pour la mise en œuvre de la méthode de prix hédonique. Doc de travail Série Méthode 05 M01, 35p. Disponible en ligne : http://www.ecologie.gov.fr

35. Thébaud, B., Corniaux, C., François, A., Powell, A., 2018. Etude sur la transhumance au Sahel (2014-2017): Dix constats sur la mobilité du bétail en Afrique de l'Ouest. 17p. www.cirad.fr.

36. Travers, M., Appéré, G., Sagot-Duvauroux, D., 2018. Prise en compte de l'hétérogénéité des préférences dans le cadre de la méthode des choix 
multi-attributs: application à un bien culturel public à vocation non touristique. Rev. Économique 69, 267-302.

37. Valbuena, D., Tui SH-K., Erenstein, O., Teufel, N., Duncan, A., Abdoulaye, T., et al. 2015. Identifying determinants, pressures and tradeoffs of crop residue use in mixed smallholder farms in Sub-Saharan Africa and South Asia. Agricultural Systems 134: 107-118. 